\title{
NOTE ON WITT GROUP AND KO-THEORY OF COMPLEX GRASSMANNIANS
}

\author{
NOBUAKI YAGITA
}

\begin{abstract}
For a complex Grassmannian $X$, there is the isomorphism between the Balmer's Witt group and the quotient of topological $K$-theories so that $W^{*}(X) \cong K O^{2 *}(X) / K U^{2 *}(X)$.
\end{abstract}

\section{INTRODUCTION}

Let $X$ be a smooth variety over a field $k$ with $1 / 2$. The Witt group $W(X)$ is the quotient of the Grothendieck group of vector bundles with quadratic forms over $X$, by subbundles $V$ with quadratic forms which admit Lagragian subbundles $E$ (i.e., $E$ is its own orthogonal complement in $V$ ).

Hence when $k=\mathbb{C}$, there is the natural map

$$
W(X) \rightarrow K O^{0}(X(\mathbb{C})) / K U^{0}(X(\mathbb{C})) .
$$

Here $K O^{0}(-)$ and $K U^{0}(-)$ is the usual (topological) real and complex $K$-theories. One purpose of this paper is to show that this map is isomorphic when $X=M_{m, n}$ the complex Grassmannian of $m$-planes in an $m+n$-plane. Moreover, we have the isomorphism

$$
W^{*}(X) \cong K O^{2 *}(X(\mathbb{C})) / K U^{2 *}(X(\mathbb{C}))
$$

where $W^{*}(X)$ is the Balmer's Witt group with $W^{0}(X)=W(X)$.

The right hand side of the above isomorphism is computed explicitly by Hara and Hara-Kono [Ha],[Ha-Ko] by using Atiyah-Hirzebruch spectral sequence, here the computation of $S q^{2}$ is most important.

On the other hand, $W^{*}(X)$ is given recently by Balmer-Calmes [Ba$\mathrm{Ca} 2$ ] in complete general forms, using $m \times n$-framed even Young diagrams. However we can also get the results (for $k=\mathbb{C}$ ) by using Pardon and Balmer-Walter spectral sequences by using the computation of $S q^{2}$ by Hara-Kono. The another purpose of this paper is to explain the relation between the results by Balmer-Calmes and HaraKono.

1991 Mathematics Subject Classification. Primary 19G12, 55N15; Secondary $55 \mathrm{~N} 20$.

Key words and phrases. Grassmannian, Witt group, $K$-theory . 
I would like to thank to Burt Totaro who suggested the above isomorphisms, to Paul Balmer who taught me the result of Calmes and him, and to Akihiro Ohshita who taught me results of Hara-Kono and him.

\section{KO-THEORY}

We explain the $K O$-theory of Garassmannian according to Hara [Ha] and Hara-Kono [Ha-Ko]. Let $M_{m, n}$ be the complex Grassmannian $G L_{m}\left(\mathbb{C}^{m+n}\right)$ of $m$-planes in $\mathbb{C}^{m+n}$. Then there is the homeomorphism

$$
M_{m+n} \cong U(m+n) /(U(m) \times U(n)) .
$$

By using the Serre spectral sequence induced from the fiber sequence

$$
U(m+n) / U(m) \times U(n) \rightarrow B U(m) \times B U(n) \rightarrow B U(m+n),
$$

we get the cohomology for any field $K$

$$
H^{*}\left(M_{m, n} ; K\right) \cong K\left[a_{1}, \ldots, a_{m}, b_{1}, \ldots, b_{n}\right] /\left(c_{1}, \ldots, c_{m+n}\right)
$$

where $a_{i}, b_{j}, c_{k}$ are Chern classes induced from maps in the above fibering, and $c_{i}=\sum a_{i-j} b_{j}$. (See also [Fu], [La] and the arguments in $\S 4$ bellow.)

Recall the coefficient ring of the (topological) $K \mathrm{O}^{*}$-theory is

$$
K O^{*} \cong \mathbb{Z}\left[\mu, \mu^{-1}, \eta, w\right] /\left(2 \eta, \eta^{3}, w^{2}-4 \mu\right)
$$

with $|\mu|=-8,|w|=-2,|\eta|=-1$. To compute $K O^{*}\left(M_{m, n}\right)$, we consider the Atiyah-Hirzebruch spectral sequence

$$
E_{2}^{*, *^{\prime}} \cong H^{*}\left(M_{m, n} ; K O^{*}\right) \Longrightarrow K O^{*}\left(M_{m, n}\right) .
$$

It is well known that the first differential is $([\mathrm{Ha}])$

$$
d_{2}(x)=\eta \otimes S q^{2}(\bar{x})
$$

where $\bar{x} \in H^{*}\left(M_{m, n} ; \mathbb{Z} / 2\right)$ is the $\bmod 2$ reduction of $x$. The Squaring operation is well known (from $\mathrm{Wu}$ formula) [Ha]

$$
S q^{2}\left(a_{1}\right)=a_{1}^{2}, \quad S q^{2}\left(a_{2 i}\right)=a_{2 i+1}+a_{2 i} a_{1} \text { for } i \geq 1 .
$$

Let $H(m, n)$ be the homology $H\left(H^{*}\left(M_{m, n} ; \mathbb{Z} / 2\right) ; S q^{2}\right)$ with the differential $S q^{2}$. Hara and Kono get this homology

Theorem 2.1. (Hara-Kono $[\mathrm{Ha}-\mathrm{Ko}])$ Let $B(k, l)$ be the graded algebra

$$
B(k, l)=\mathbb{Z} / 2\left[a_{2}^{2}, \ldots, a_{2 k}^{2}, b_{2}^{2}, \ldots, b_{2 l}^{2}\right) /\left(c_{2}^{2}, \ldots, c_{2 k+2 k}^{2}\right) .
$$

Then we have the isomorphism

$$
H(m, n) \cong\left\{\begin{array}{l}
B(k, l) \quad \text { if }(m, l)=(2 k, 2 k),(2 k+1,2 l),(2 k, 2 l+1) \\
B(k, l) \oplus B(k, l)\left\{a_{2 k+1} b_{2 l}\right\} \quad \text { if }(m, l)=(2 k+1,2 l+1) .
\end{array}\right.
$$


They also proved

Theorem 2.2. ([Ha-Ko]) The Atiyah-Hirebruch spectral sequence collapses from $E_{3}^{*, *^{\prime}}$-term.

For the proof of this theorem, Hara and Kono used the natural maps $U(n) \rightarrow S p(n)$ and $S p(n) \rightarrow U(2 n)$. Let us write $N_{m, n}=S p(m+$ $n) / S p(m) \times S p(n)$ and consider the maps

$$
M_{m, n} \stackrel{q}{\rightarrow} N_{m, n} \stackrel{c^{\prime}}{\rightarrow} M_{2 m, 2 n} .
$$

The cohomology is also computed as the case $U(n)$,

$$
H^{*}\left(N_{n, m} ; \mathbb{Z} / 2\right) \cong \mathbb{Z} / 2\left[q_{1}, \ldots, q_{m}, r_{1}, \ldots, r_{n}\right] /\left(s_{1}, \ldots, s_{m+n}\right),
$$

$s_{i}=\sum q_{i-j} r_{j}$ with $q^{*} q_{i}=a_{i}^{2}$ and $c^{\prime *} a_{2 i}=q_{i}\left(c^{\prime *}\left(a_{2 i-1}\right)=0\right)$.

Note $K O^{\text {odd }}=K O^{8 *-1}$ and $H^{*}\left(N_{m, n}\right)=H^{4 *}\left(N_{m, n}\right)$. By the dimensional reason for differntial $\operatorname{deg}\left(d_{r}\right)=(r,-r+1)$, we know the AtiyahHirzebruch spectral sequence for $K O^{*}\left(N_{m, n}\right)$ collapses from $E_{2}^{*, *^{\prime}}$-term, that means

$$
\operatorname{grKO} O^{*}\left(N_{m, n}\right) \cong K O^{*} \otimes H^{*}\left(N_{m, n} ; \mathbb{Z}\right) .
$$

Proof of Theorem 2.2. By the naturality of the spectral sequence, the maps $q^{*}, c^{\prime *}$ are defined as maps of spectral sequences. The fact $q^{*} q_{i}=$ $a_{i}^{2}$ implies that $d_{r}\left(a_{i}^{2}\right)=0$ for all $r>1$. The fact $c^{\prime *} a_{2 i}=q_{i}$ implies that $a_{2 i}^{2} \neq 0$ in $E_{\infty}^{*, *^{\prime}}$ and moreover each nonzero element in $B(k, l)$ is also nonzero in $E_{\infty}^{*, *^{\prime}}$. If $d_{r}\left(a_{2 k+1} b_{2 l}\right) \neq 0$, then it is contained in $B(k, l)$ by dimensional reason ; this is a contradiction to the preceding result.

There is the well known exact sequence for topological space $X$

$$
(1) \quad \rightarrow K O^{*}(X) \stackrel{\eta}{\rightarrow} K O^{*}(X) \stackrel{c}{\rightarrow} K U^{*}(X) \stackrel{r}{\rightarrow} K O^{*}(X) \rightarrow
$$

where $c$ is the complexification and $r$ is the restriction maps. Therefore

$$
K O^{*}(X) / K U^{*}(X) \cong \eta K O^{*}(X) .
$$

From the above theorem, we see

$$
E_{\infty}^{o d d, *} \cong E_{\infty}^{8 *-1, *} \cong \mathbb{Z} / 2\{\eta\}\left[\mu, \mu^{-1}\right] \otimes H(m, k) .
$$

Hence we have

Corollary 2.3. $\operatorname{gr} K O^{2 *}\left(M_{m, n}\right) / K U^{2 *}\left(M_{m, n}\right) \cong H(m, n)$. 


\section{BALMER's Witt Group.}

For a smooth $X$ over a field $k$ with $1 / 2 \in k$, let $W(X)$ denote the Witt group of $X$. Balmer defined the periodic Witt group $W^{i}(X) \cong$ $W^{i+4}(X),(i \in \mathbb{Z})$ with $W^{0}(X)=W(X)$.

Balmer and Walter [Ba-Wa] define the Gersten-Witt complex

$$
0 \rightarrow W(k(X)) \rightarrow \oplus_{x \in X^{(1)}} W(k(x)) \rightarrow \ldots \oplus_{x \in X^{(n)}} W(k(x)) \rightarrow 0 .
$$

Let $H^{*}(W(X))$ denote the cohomology group of the above cochain complex, with $W(k(X))$ places in degree 0. From the above complex, we have the (Balmer-Walter) spectral sequence

$$
E(B W)_{2}^{r, s} \cong\left\{\begin{array}{l}
H^{r}(W(X))\left(s=4 s^{\prime}\right) \quad \Longrightarrow W^{r+s}(X) \\
0 \quad(s \neq 0(\bmod (4))
\end{array} .\right.
$$

By the affirmative answer of the Milnor conjecture of quadratic forms by Orlov-Vishik-Voevodsky [Or-Vi-Vo], we have the isomorphism of graded rings $H^{*}(k(x) ; \mathbb{Z} / 2) \cong g r W^{*}(k(x))$. Using this fact, Pardon $([\mathrm{Pa}],[\mathrm{To}])$ defined the spectral sequence

$$
E(P)_{2}^{r, s} \cong H_{Z a r}^{r}\left(X ; H_{\mathbb{Z} / 2}^{s}\right) \Longrightarrow H^{r}(W(X)) \cong E(B W)_{2}^{r, 4 s}
$$

so that the differential $d_{r}$ has degree $(1, r-1)$ for $r \geq 2$. Here $H_{\mathbb{Z} / 2}^{n}$ the Zarisky sheaf induced from the presheaf $H_{e t}^{n}(V ; \mathbb{Z} / 2)$ for open subset $V$ of $X$.

The above sheaf cohomology $H_{Z a r}^{r}\left(X ; H_{\mathbb{Z} / 2}^{s}\right)$ relates the motivic cohomology $H^{*, *^{\prime}}(X ; \mathbb{Z} / 2)\left(\right.$ see $\left[\right.$ Vo1-3]). Let $\tau \in H^{0,1}(\operatorname{Speck}(k) ; \mathbb{Z} / 2) \cong$ $\mathbb{Z} / 2$ be a generator. (Hence $H^{*, *^{\prime}}(\operatorname{Speck}(\mathbb{C}) ; \mathbb{Z} / 2) \cong \mathbb{Z} / 2[\tau]$.) Then we get the long exact sequence from the solution of Beilinson-Lichtenbaum conjecture by Voevodsky [Or-Vi-Vo]

$$
\begin{aligned}
\rightarrow & H^{m, n-1}(X ; \mathbb{Z} / 2) \stackrel{\times \tau}{\rightarrow} H^{m, n}(X ; \mathbb{Z} / 2) \\
& \rightarrow H_{Z a r}^{m-n}\left(X ; H_{\mathbb{Z} / 2}^{n}\right) \rightarrow H^{m+1, n-1}(X ; \mathbb{Z} / 2) \stackrel{\times \tau}{\rightarrow} .
\end{aligned}
$$

Therefore, we have

Lemma 3.1. $E(P)_{2}^{m-n, n} \cong H_{Z a r}^{m-n}\left(X ; H_{\mathbb{Z} / 2}^{n}\right) \cong$

$$
H^{m, n}(X ; \mathbb{Z} / 2) /(\tau) \oplus \operatorname{Ker}(\tau) \mid H^{m+1, n-1}(X ; \mathbb{Z} / 2) .
$$

In particular, $E(P)_{2}^{m, m} \cong H^{2 m, m}(X ; \mathbb{Z} / 2) \cong C H^{m}(X) / 2$. Moreover Totaro proved

Lemma 3.2. (Totaro [To]) If $x \in E(P)_{2}^{m, m} \cong C H^{m}(X) / 2$, then $d_{2}(x)=S q^{2}(x)$. 
Now we consider the case $X=M_{m, n}$ and $k=\mathbb{C}$. Since $M_{m, n}$ is cellular, we see

$H^{2 *, *^{\prime}}\left(M_{m, n} ; \mathbb{Z} / 2\right) \cong \mathbb{Z} / 2[\tau] \otimes C H^{*}\left(M_{m, n}\right) / 2 \cong \mathbb{Z} / 2[\tau] \otimes H^{2 *}\left(M_{m, n} ; \mathbb{Z} / 2\right)$.

Hence we have

$$
E(P)_{2}^{*, *^{\prime}} \cong E(P)_{2}^{* * *} \cong H^{2 *}\left(M_{m, n} ; \mathbb{Z} / 2\right) .
$$

From the result of Totaro, we have

$$
E(P)_{3}^{*, *^{\prime}} \cong H\left(H^{2 *}\left(M_{m, n} ; \mathbb{Z} / 2\right) ; S q^{2}\right)=H(m, n) .
$$

By dimensional reason of differential $\operatorname{deg}\left(d_{r}\right)=(1, r-1)$, it is immediate that the spectral sequence collapses from $E_{3}^{*, *^{\prime}}$-term, i.e., $E(P)_{3}^{*, *^{\prime}} \cong$ $E_{\infty}^{*, *^{\prime}}$.

The Balmer-Walter spectral sequence also collapses, that is, we will prove

$$
E(P)_{\infty}^{*, *} \cong E(B W)_{2}^{*, r} \cong E(B W)_{\infty}^{*, r}, \quad r=0 \bmod (4) .
$$

For this we consider the $S p(n)$-version of above arguments. We consider spectral sequences for $N_{m, n}$. By dimensional reason, the Pardon and Balmer-Walter spectral sequences collapse from $E_{2}$-terms. (Note $H^{*}\left(N_{m, n} ; \mathbb{Z} / 2\right)=0$ for $* \neq 0 \bmod (4)$. $)$ That is

$$
g r W^{*}\left(M_{m, n}\right) \cong E(B W)_{\infty}^{*, r} \cong H^{2 *}\left(N_{m, n} ; \mathbb{Z} / 2\right) .
$$

Then the arguments of the proof of Theorem 2 also work. Thus we see the collapseness of the Balmer-Walter spectral sequence for $M_{m, n}$. Therefore we have isomorphisms

\section{Theorem 3.3.}

$$
g r W^{*}\left(M_{m, n}\right) \cong H(m, n) \cong g r K O^{2 *}\left(M_{m, n}\right) / K U^{2 *}\left(M_{m, n}\right) .
$$

\section{Young Diagram AND Witt Group}

In this section we recall the result of Balmer-Calmes [Ba-Ca1,2], and consider relation to the result of Hara-Kono.

The cohomology $H^{*}\left(M_{m, n}\right)$ (or $C H^{*}\left(M_{m, n}\right)$ ) is also computed by induction on $n, m$. In fact the following exact sequence

$$
\rightarrow H^{*-\left|a_{n}\right|}\left(M_{m, n-1}\right) \stackrel{g_{*}}{\rightarrow} H^{*}\left(M_{m, n}\right) \stackrel{f^{*}}{\rightarrow} H^{*}\left(M_{m-1, n}\right) \stackrel{\partial}{\rightarrow}
$$

becomes split since $\partial=0$. Here $g_{*}$ is the Gysin map for the embedding $M_{m, n-1} \subset M_{m, n}$. The map $f^{*}$ is induced from

$$
M_{m-1, n} \stackrel{\text { proj. }}{\longleftarrow} M_{m, n}-M_{m, n-1} \subset M_{m, n} .
$$

(See $[\mathrm{Ba}-\mathrm{Ca} 1,2]$ or Laksov $[\mathrm{La}],[\mathrm{Fu}])$. Here note $g_{*}(x)=a_{m} \cdot x$. 
It is well known that the cohomology of $M_{m, n}$ is stated also by using Young diagram. The $m \times n$-framed partition $\lambda=\left(\lambda_{1}, \ldots, \lambda_{d}\right)$ means

$$
n \geq \lambda_{1} \geq \ldots \geq \lambda_{d} \geq 1 \text { and } m \geq d .
$$

The partition $\lambda$ corresponds a Young diagram, consisting of $\lambda_{i}$ boxes in the $i$-th row from the top, lined up on the left. Then $m \times n$-framed Young diagrams with $d=|\lambda|=\lambda_{1}+\ldots+\lambda_{d}$ form the basis of $H^{d}\left(M_{m, n}\right)$, namely, $H^{*}\left(M_{m, n}\right) \cong \oplus_{*=|\lambda|} \mathbb{Z}$. This fact is shown as follows.

The Young diagram for the conjugate partition $\tilde{\lambda}$ of $\lambda$ is obtained by interchanging rows and columns in the diagram. For a Young diagram $\lambda$, we can define the Schur polynomial (e.g. see $[\mathrm{Fu}]$ ) by

$$
\Delta_{\lambda}(b)=\operatorname{det}\left(b_{\lambda_{i}+j-i}\right) \in H^{*}\left(M_{m, n}\right) .
$$

It is known (Lemma 14.5.1 in $[\mathrm{Fu}]) \Delta_{\lambda}(b)=\Delta_{\tilde{\lambda}}(a)$. Hence we have $\Delta_{(k)}(b)=b_{k}$ and $\Delta_{(\tilde{k})=(1, \ldots, 1)}(b)=a_{k}$. Moreover we see by the above definition of $\Delta_{\lambda}$,

$$
\Delta_{\lambda}(b)=b_{\lambda_{1}} \ldots b_{\lambda_{d}} \bmod \left(F_{>\lambda}\right) .
$$

Here $F_{>\lambda}$ is the filtration of elements $b_{\lambda^{\prime}{ }_{1} \ldots .} b_{\lambda^{\prime}{ }_{d^{\prime}}}$ with $\lambda^{\prime}>\lambda$ by the lexicographical order.

We still know the above $\Delta_{\lambda}(b)$ make a basis $[\lambda]$ of $H^{*}\left(M_{m, n}\right)$ from (2.1). However we can also get it by induction by using the short exact sequence (4.1) such that

$$
g_{*}([\lambda])=[(1, \ldots, 1)+\lambda]=\left[\left(1+\lambda_{1}, \ldots, 1+\lambda_{d}, 1, \ldots, 1\right)\right],
$$

(Indeed, $\Delta_{g_{*}(\lambda)}(b)=a_{m} \cdot \Delta_{\lambda}(b) \bmod \left(F_{>g^{*}(\lambda)}\right)$.) The induced map $f^{*}$ is given $f^{*}(\lambda)=\lambda$ for $d<m$, and $=0$ for $d=m$.

Let us say that framed Young diagram $\lambda$ is strongly even if all its segments have even length, namely all $\lambda_{i}, \tilde{\lambda}_{i}$ are even. Then its Schur polynomial is written

$$
\Delta_{\lambda}(b)=\left(b_{\lambda_{1}}^{2} b_{\lambda_{3}}^{2} \ldots b_{\lambda_{d-1}}^{2}\right) \quad \bmod \left(F_{>\lambda}\right) .
$$

Hence if $m$ or $n$ is even, then set of strongly even $m \times n$-framed diagrams make $\mathbb{Z} / 2$-base of the ring $B(k, l)$ given in Theorem 2.1 by Hara-Kono.

Balmer and Calmes results generalize above arguments. We can consider the generalized Witt group $W^{i}(X ; L)$ for $i \in \mathbb{Z} / 4$ and $L \in$ $\operatorname{Pic}(X) / 2$ such that the usual Witt group $W^{i}(X)=W^{i}\left(X ; O_{X}\right)$.

Let us say that framed Young diagram $\lambda$ is even if all its segments have even length, which are strictly inside of the frame, namely all $\lambda_{i}-\lambda_{i+1}$ for $1 \leq i \leq d-1, \tilde{\lambda}_{i}-\tilde{\lambda}_{i+1}$ for $1 \leq i \leq \tilde{d}-1$ are even. Let $t(\lambda)$ be half of the perimeter of $\lambda$. 
Theorem 4.1. (Balmer-Camles [Ba-Ca2]) The total Witt group

$$
W^{\text {tot }}\left(M_{m, n}\right)=\oplus_{i \in \mathbb{Z} / 4, L \in \mathbb{Z} / 2} W^{i}\left(M_{m, n} ; L\right)
$$

has $\mathbb{Z} / 2$-basis indexed by even Young diagrams $\lambda$. The corresponding base $[\lambda]$ is in $W^{|\lambda|}\left(M_{m, n}, t(\lambda)\right)$.

Remark. In [Ba-Ca2], the theorem is stated in very generalized situation.

Let $m$ or $m$ be even. Then when $t(\lambda)=0$, it is easily seen that $\lambda$ is even means strongly even. So the argument before the above theorem explains the relation of the results by Hara-Kono and Balmer-Calmes.

Next we consider the case $(m, n)=(2 k+1,2 l+1)$. Each even $m \times n$ framed diagram $\lambda$ with $t(\lambda)=0$ is easily seen strongly even $\left[\lambda^{s e}\right]$ or $\left[\Gamma \lambda^{s e}\right]$ which is defined as

$$
\left[(2 l+1,1, \ldots, \stackrel{m}{1})+\left(0, \lambda^{s e}\right)\right]=\left[\left(2 l+1, \lambda_{1}^{s e}+1, \ldots, \lambda_{d^{s e}}^{s e}+1,1 \ldots, \stackrel{m}{1}\right)\right] .
$$

Note $\mu=(2 l+1,1, . ., 1)$ is even but not strongly even, and $|\mu|=o d d$, $t(\mu)=0$. We still know $\left[\lambda^{s e}\right]$ form the $\mathbb{Z} / 2$-basis of $B(k, l)$ in Theorem 2.1. Note that

$$
\Delta_{\mu}(b)=b_{2 l+1} a_{2 k}=a_{2 k+1} b_{2 l} \bmod \left(F_{>\mu}\right) .
$$

Hence $\left[\Gamma \lambda^{s e}\right]$ form a basis of $B(k, l)\left\{a_{2 k+1} b_{2 l}\right\}$. Therefore we see even $m \times n$-framed Young diagrams form the base of

$$
B(k, l) \oplus B(k, l)\left\{a_{2 k+1} b_{2 l}\right\}=H(m, n) .
$$

Thus we can explain the relation between Hara-Kono and BalmerCalmes.

Balmer and Camles prove their theorem by showing following (long) exact sequence. (They construct the Gysin and the boundary maps as the maps in $W^{\text {total }}(X)$.) Let $g: Z \subset X$ be a regular closed immersion of codim $=c \geq 2$, and $U=X-Z$. Let $\omega_{g}$ be the relative canonical bundle (for the definition see [Ba-Ca1]). Then there is the natural exact sequence

$$
\rightarrow W^{*-c}\left(Z,\left.\omega_{g} \otimes L\right|_{Z}\right) \stackrel{g_{*}}{\rightarrow} W^{*}(X, L) \stackrel{f^{*}}{\rightarrow} W^{*}\left(Z,\left.L\right|_{Z}\right) \stackrel{\partial}{\rightarrow} .
$$

In general $\partial \neq 0$. In fact, when $X=M_{m, n}$, it is proved (Figures 4-6 in $[\mathrm{Ba}-\mathrm{Ca} 2])$.

$$
\begin{aligned}
& g_{*}([\lambda])=\left\{\begin{array}{l}
{\left[\left(1, . .,{ }^{m}\right)+\lambda\right] \text { if } m-d: \text { even }} \\
0 \text { otherwise, }
\end{array} f^{*}([\lambda])=\left\{\begin{array}{l}
{[\lambda] \text { if } d<m} \\
0 \text { otherwise, }
\end{array}\right.\right. \\
& \partial([\lambda])=\left\{\begin{array}{l}
{[\lambda-(1, \ldots, \stackrel{d}{1})] \text { if } \lambda_{d}: \text { odd }} \\
0 \text { otherwise. }
\end{array}\right.
\end{aligned}
$$


In the $\mathbb{A}^{1}$-homotopy category, Hornbostel $[\mathrm{Ho}]$ proved that $W^{*}(-)$ is represented as a $\mathbb{P}^{1}$-spectrum. This implies

$$
W^{*+1}\left(\mathbb{P}^{1} \wedge X\right) \cong W^{*}(X) .
$$

Therefore we can define the natural map

$$
q: W^{*}(X) \rightarrow K O^{2 *}(X(\mathbb{C})) / K U^{2 *}(X(\mathbb{C})) \text { for all } *
$$

We will see that this map induces the isomorphism given preceding and this sections.

First we consider the cases $(m, n)=(2 k, 2 l)$. Let $\tilde{g}: M_{m, n-2} \subset M_{m, n}$ and $\tilde{f}: M_{m, n} \rightarrow M_{m-2, n}$. (Note $\tilde{g}_{*}(x)=a_{m}^{2} x$.) Then we have the commutative diagram.

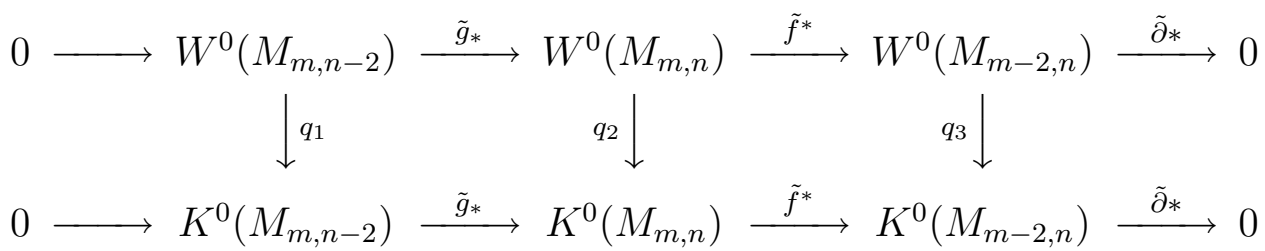

where $K^{*}(X)=K U^{*}(X) / K O^{*}(X)$. The exactness of rows follow from the isomorphism given in the preceding or this sections. (In fact this case $W_{m, n}^{*}=W_{m, n}^{0}$.) By the induction and five lemma, we have the isomorphism of $q_{2}$. The case $m$ or $n$ even follows from the above result and the naturality.

The case $(m, n)=(2 k+1,2 l+1)$ is proved as follows. Let $g^{\prime}$ : $M_{2 k, 2 l} \rightarrow M_{2 k+1,2 l+1}$ and recall $g_{*}^{\prime}([\lambda])=[\Gamma \lambda]$. Consider the following diagram

$$
\begin{gathered}
W^{0}\left(M_{2 k, 2 l}\right) \stackrel{g^{\prime}}{\cong} W^{1}\left(M_{2 k+1,2 l+1}\right) \\
\qquad q_{1} \\
K^{0}\left(M_{2 k, 2 l}\right) \stackrel{g_{*}^{\prime}}{\stackrel{q_{2}}{\longrightarrow}} K^{2}\left(M_{2 k+1,2 l+1}\right) .
\end{gathered}
$$

We see $q_{2}$ is isomorphic for this case. Similarly we consider $f^{\prime}$ : $M_{2 k, 2 l} \rightarrow M_{2 k+1,2 k+1}$. By the isomorphism of preceding or this section, we have the isomorphism $f^{\prime *}: W^{0}\left(M_{2 k+1,2 k+1}\right) \cong W^{0}\left(M_{2 k, 2 k}\right)$. This also induces the isomorphism of $q$ for $W^{0}\left(M_{m, n}\right) \rightarrow K^{0}\left(M_{m, n}\right)$. Thus we can show

Theorem 4.2. The map $q: W^{*}\left(M_{m, n}\right) \rightarrow K O^{2 *}\left(M_{m, n}\right) / K U^{2 *}\left(M_{m, n}\right)$ induces the isomorphism.

Corollary 4.3. There is the isomorphism of graded rings

$$
g r W^{*}\left(M_{m, n}\right) \cong H(m, n) .
$$


In particular, we note that $g r W^{0}\left(M_{2 k, 2 l}\right) \cong H^{*}\left(M_{k, l} ; \mathbb{Z} / 2\right)$.

\section{REFERENCES}

[Ba-Ca1] P.Balmer and B.Calmes. Geometric description of the connecting homomorphism for Witt groups. www.math.uiuc.edu/K-theory/902 (2008).

[Ba-Ca2] P.Balmer and B.Calmes. Witt groups of Grassmann varieties. www.math.uiuc.edu/K-theory/903 (2008).

[Ba-Wa] P.Balmer and C.Walter. A Gersten-Witt spectral sequence for regular schemes. Ann.Scient.Éc.Norm.Sup. 35 (2002) 127-152.

[Fu W. Fulton. Intersection theory, second ed. Ergebnisse der Mathematic und ihrer Grenzgebiete. 3 Springer-Verlag, Berlin. (1998).

[Ha] S. Hara. Note on $K O$-theory of $B O(n)$ and $B U(n)$. J. Math. Kyoto Univ. 31 (1991) 487-493.

[Ha-Ko] S. Hara and A.Kono. KO-theory of complex Grassmannian. J. Math. Kyoto Univ. 31 (1991) 827-833.

[Ho] J. Hornbostel. $\mathbb{A}^{1}$-representability of hermitian $K$-theory and Witt group. Toplogy. 44 (2005) 661-687.

[La] D.Laksov. Algebraic cycles on Grassmann varieties. Adv. in Math. 9 (1972) 267-295.

[Oh] A.Ohshita. On KO-theory of flag manifolds of exceptional Lie groups. preprint. (2008).

[Or-Vi-Vo] D. Orlov, A. Vishik and V. Voevodsky. An exact sequence for Milnor's K-theory with applications to quadric forms. Ann of Math. 165 (2007), $1-13$.

[Pa] W.Pardon. The filtered Gersten-Witt complex for regular schemes. www.math.uiuc.edu/K-theory/0419 (2000).

[To] B. Totaro. Non-injectivity of the map from the Witt group of a variety to the Witt group of its function field. J. Inst. Math. Jussieu 2 (2003), 483-493.

[Vo1] V. Voevodsky. The Milnor conjecture. www.math.uiuc.edu/Ktheory/0170 (1996).

[Vo2] V.Voevodsky. Reduced power operations in motivic cohomology. Publ.Math. IHES. 98 (2003), 1-54.

[Vo3] V.Voevodsky. Motivic cohomology with $\mathbb{Z} / 2$-coefficients. Publ.Math. IHES. 98 (2003), 59-104.

[Ya] N. Yagita. Coniveau spectral sequence and motivic cohomology of quadrics and classifying spaces. www.math.uiuc.edu/K-theory/0709 (2004).

Department of Mathematics, Faculty of Education, Ibaraki UniverSITY, Mito, IBARAKI, JAPAN

E-mail address: yagita@mx.ibaraki.ac.jp 